\title{
Transition of a microRNA from Repressing to Activating Translation Depending on the Extent of Base Pairing with the Target
}

\author{
Ashesh A. Saraiya, Wei Li, Ching C. Wang* \\ Department of Pharmaceutical Chemistry, University of California San Francisco, San Francisco, California, United States of America
}

\begin{abstract}
MicroRNAs are major post-transcriptional regulators of gene expression. Here we show in the ancient protozoan Giardia lamblia a snoRNA-derived 26-nucleotide microRNA, miR3, which represses the translation of histone H2A mRNA containing an imperfect target but enhances translation when the target is made fully complementary. A stepwise mutational analysis of the fully complementary target showed that the activating effect of miR3 was significantly reduced when a single nucleotide at the $5^{\prime}$-end of the target was altered. The effect of miR3 became repressive when 12 of the nucleotides lost their complementation to miR3 with maximum repression reached when only 8 base-pairs remained between the miR3 seed sequence and the target. A synthetic 8-nucleotide RNA oligomer of the miR3 seed sequence was found capable of exerting a similar Argonaute-dependent translational repression. This is the first report showing a correlation between the extent of base-pairing with the target and a change in miRNA function.
\end{abstract}

Citation: Saraiya AA, Li W, Wang CC (2013) Transition of a microRNA from Repressing to Activating Translation Depending on the Extent of Base Pairing with the Target. PLoS ONE 8(2): e55672. doi:10.1371/journal.pone.0055672

Editor: K.T. Jeang, National Institute of Health, United States of America

Received October 4, 2012; Accepted December 29, 2012; Published February 6, 2013

Copyright: (c) 2013 Saraiya et al. This is an open-access article distributed under the terms of the Creative Commons Attribution License, which permits unrestricted use, distribution, and reproduction in any medium, provided the original author and source are credited.

Funding: This work was supported by the National Institutes of Health [AI30475 to CCW]. The funders had no role in study design, data collection and analysis, decision to publish, or preparation of the manuscript.

Competing Interests: The authors have declared that no competing interests exist.

*E-mail: ccwang@cgl.ucsf.edu

\section{Introduction}

MicroRNAs (miRNAs) are small, non-coding RNAs involved in post-transcriptional gene regulation. The action is mediated through base-pairings between an Argonaute protein-complexed miRNA and an imperfectly matched target site usually located at the 3 '-end of a target mRNA [1,2], resulting in translational repression followed by deadenylation and degradation of the mRNA [3,4]. Approximately $1 \%$ of the genes in animals have been found to encode miRNAs, which are often highly conserved across a wide range of species [1].

Since the original discovery that miRNAs were essential regulators of development in Caenorhabditis elegans [5], there have been increasing numbers of examples indicating that miRNAs are involved in many divergent cellular processes. To cite but a few examples, they modulate hematopoietic lineage differentiation [6], regulate brain morphogenesis in zebrafish [7] and induce cell proliferation [8] and apoptosis in Drosophila [9]. Variations in miRNA expression levels are thus often associated with various types of tumors [10,11]. Viruses often use miRNAs in controlling their host cells [12], whereas the host also use miRNAs to block essential viral functions [13]. miRNAs may thus constitute an important new layer of regulation of gene expression in many eukaryotes.

Rules for miRNA-target interactions are still unclear, but some general guidelines have been established. Base-pairings of the 5' end of miRNA at positions 2-8, the seed sequence, with the target is usually crucial for miRNA targeting. The duplex thus formed has been postulated to function as a nucleation site for the remainder of the miRNA-target interaction, though experimental support for such hypothesis is still lacking [1,14,15]. The $3^{\prime}$ sequence downstream from the seed sequence of a miRNA, however, often forms imperfect base-pairings containing mismatches, GU wobbles and bulges with the target. These imperfections in complementation have been routinely identified among miRNA-target complexes, suggesting that perfect basepairings are not necessary for miRNA function $[1,14,15]$. Comparative analysis of miRNAs in Drosophila [14] and mammals [16] indicated that the 3 ' - pairing of functional miRNAs to their targets are no more than expected by chance. Mammalian targets for miRNAs can be readily predicted around the $3^{\prime}$ untranslated regions (UTRs) of transcripts by searching for conserved matches to the 7-nucleotide (nt) seed sequences in miRNAs [16].

Though an imperfectly matched miRNA-mRNA pair with central bulges leads to translational inhibition followed by deadenylation and degradation of the mRNA [3,4], a perfectly complementary pair tends to promote mRNA slicing by the Argonaute protein $[17,18]$. There are, however, occasional exceptions to this rule $[19,20]$, which could be attributed to the presence of Argonaute proteins lacking endonucleolytic activity [21]. Loading of a miRNA onto such a protein could lead to only translational repression of the target mRNA.

Several functional miRNAs have been recently identified in Giardia lamblia, a deeply branched protozoan parasite responsible for the common diarrheal disease giardiasis [22,23,24,25]. These miRNAs are derived from either snoRNAs or by a canonical biogenetic pathway mediated by the Giardia Dicer protein, GIDcr 
$[22,23,24,25]$. The miRNAs range in size from 26 to $28 \mathrm{nts}$, which is in good agreement with the size estimated from the crystal structure of GIDcr [26]. There is a single Argonaute protein (GlAgo) in Giardia. It forms complexes with the miRNAs and mediates their actions on mRNAs carrying imperfectly complementary target sites by repressing mRNA translation without apparent degradation. Reporter mRNAs with fully complementary target sites to miR4 [24] and miR5 [22] were tested previously and were found to be translationally repressed without mRNA degradation, suggesting that GlAgo may lack endonucleolytic activity and the miRNA action in Giardia may be confined to translational repression regardless of the extent of base-pairings between the miRNAs and their targets (see Discussion).

Here we report in Giardia another snoRNA-derived miRNA, miR3, that has a $26 \mathrm{nt}$ sequence and represses translation of a histone $\mathrm{H} 2 \mathrm{~A}$ mRNA carrying an imperfectly complementary target site by $\sim 20 \%$. It has similar features to all the other miRNAs previously identified in Giardia [22,23,24,25]. However, when we altered the target site sequence to fully complement miR3, the latter showed an unexpectedly strong activating effect on translation of the mRNA. A stepwise mutational analysis of the target sequence indicated that a full $26 \mathrm{nt}$ complementation with miR 3 is required for maximum activation, whereas base-pairing with just the 8-nt seed sequence of miR3 results in maximum repression. Both actions are dependent on the presence of GlAgo.

This is, by our knowledge, a novel observation, which may help us further understand the mechanism behind miRNA regulation of gene expression. The dramatic transition from repressing to activating translation with increased base-pairings between the miRNA and the target may provide a good opportunity to study the detailed mechanisms of miRNA-mediated regulation of mRNA translation.

\section{Results}

\section{miR3 is a snoRNA-derived miRNA}

miR3 was originally identified during the cloning of size fractionated small RNAs from Giardia trophozoites [25]. It has a length of 26 nts (5' GCA GAC AAC GCA UCA CGG CUC UGA CG $3^{\prime}$ ), is derived from the $3^{\prime}$-end of a conserved orphan Box C/D snoRNA, GlsR 16, and requires the GlDcr for processing into the mature form [25]. To complete the characterization of this small RNA, we used fluorescence in situ hybridization (FISH) to indicate that miR3 is primarily localized to the cytoplasm while GlsR16 is concentrated in the nucleolus-like organelles of Giardia trophozoites (Figure S1A). It confirms that GlsR16 is most likely a snoRNA localized to the nucleolus and that miR3 is a cytoplasmic product derived from GlsR 16 .

A 2xHA tagged Giardia Argonaute (HA-GlAgo) protein expressed in Giardia trophozoites was co-immunoprecipitated with a small RNA band of $\sim 26-30$ nts $[22,24]$. Using a $5^{\prime}$ nuclease qPGR assay (Taqman) (Figure S1B), the presence of miR 3 in the GlAgo-associated small RNA band was demonstrated, suggesting that miR3 could be a functional miRNA (Figure S1B).

\section{Identification of the Putative Targets of miR3}

We used the miRanda (version 3) program to identify potential targets of miR3 in the Giardia genomic database [27]. Fifty nts upstream and $100 \mathrm{nts}$ downstream of the stop codon from each open reading frame (ORF) in the Giardia database were chosen for the search [28]. The resulting hits were further filtered to remove targets with less than 5 complementary bases in the seed sequence while allowing one G:U wobble pair. Of the 4969 ORFs in the Giardia genome database (version 1.1), 127 genes were found to carry potential miR3 targets (Table S1), of which 55 encode annotated proteins while the remaining 72 encode for hypothetical proteins. Among the former, there were two variant-specific surface proteins (VSP), one high cysteine membrane protein and a spectrum of other proteins without any apparent correlation among their biological functions (Table S1). This is in contrast to the other previously characterized Giardia miRNAs, which act on large numbers of VSP transcripts [23,24,25]. No target with complete complementarity to miR 3 was identified. We chose three putative targets of miR3 for further analysis based on their relatively high miRanda scores and low free energies of miR3 binding. All three target sites are positioned across the ORF and the 3'-UTR of the gene. These sites include a potassiumtransporting ATPase alpha chain 1, a Ser/Thr phosphatase 2A $65 \mathrm{kD}$ regulatory subunit $\mathrm{A}$ and histone $\mathrm{H} 2 \mathrm{~A}$ (Table 1). Two copies of each of the potential target sites were inserted into the 3 ' UTR of a Renilla luciferase (RLuc) reporter transcript, introduced into Giardia trophozoites together with chemically synthesized miR3 by electroporation, and assayed for RLuc expression (Figure 1A). The potassium-transporting ATPase target site (RL-PTA-TS) has the most extensive base pairings with miR3 (Table 1), but RLuc expression $(\sim 3 \%)$ was not appreciably affected by miR3 (Figure 1A). Increased repression of RLuc activity $(\sim 15 \%)$ was observed for the Ser/Thr phosphatase 2A (RL-P2A-TS) target site, whereas the histone H2A (RL-H2A-TS) target site with the lowest miRanda score (Table 1) showed the highest repression by $\operatorname{miR} 3(\sim 18 \%)$ (Figure 1A).

\section{Enhanced Expression of a Reporter Transcript Carrying a Fully Complementary Target Site for miR3}

This somewhat unexpected data in Figure 1A appeared to suggest that the extent of sequence matching between miR 3 and its target does not necessarily dictate the efficiency of miR3 inhibition of RLuc expression. To test this possibility, we incorporated two $26 \mathrm{nt}$ target sites fully complementary (FG) to miR3 into the RLuc transcript 3 '-UTR (RL-FG) and tested if miR3 would have any effect on its expression in Giardia. The results presented in Figure $1 \mathrm{~B}$ indicate that, instead of an inhibitory effect, miR3 enhanced the expression of RL-FG by as much as two-fold. There was no significant change in the level of RL-FC transcript when co-transfected with miR3 (Figure S2B), suggesting that miR3 enhances the translation of the RL-FC transcript. The experiment was then repeated in a Giardia mutant cell line (GlAgo KD), in which the GlAgo protein was reduced by a giardiavirus-mediated antisense-hammerhead ribozyme knockdown of the GlAgo mRNA [25]. The experimental outcome showed that miR3 enhanced expression of RL-FC by only $\sim 26 \%$ in these cells (Figure 1B), indicating that the miR3-mediated enhancement of translation is dependent on GlAgo. To further verify whether the stimulatory effect of miR3 is through hybridizing to the fully complementary target, a 2' O-methyl antisense oligo of miR3 (ASO) was co-introduced with miR3 into the Giardia cells. The ASOs are known to form stable duplexes with the corresponding miRNAs to block their functions [29]. The introduction of an equivalent amount of ASO with miR3 into Giardia resulted in virtually no effect on the expression of RL-FC (Figure 1C). When only the ASO was introduced, there was a $\sim 25 \%$ reduction in $\mathrm{RL}-\mathrm{FG}$ expression, suggesting the presence of endogenous miR3 in Giardia is capable of enhancing the expression of RL-FC by 25\% (Figure 1C).

Enhancement of histone $\mathrm{H} 2 \mathrm{~A}$ gene expression carrying a target site fully complementary to miR3. The enhanced expression of a reporter transcript by miR 3 prompted us to look at the potential effect of miR3 on the expression of an endogenous 

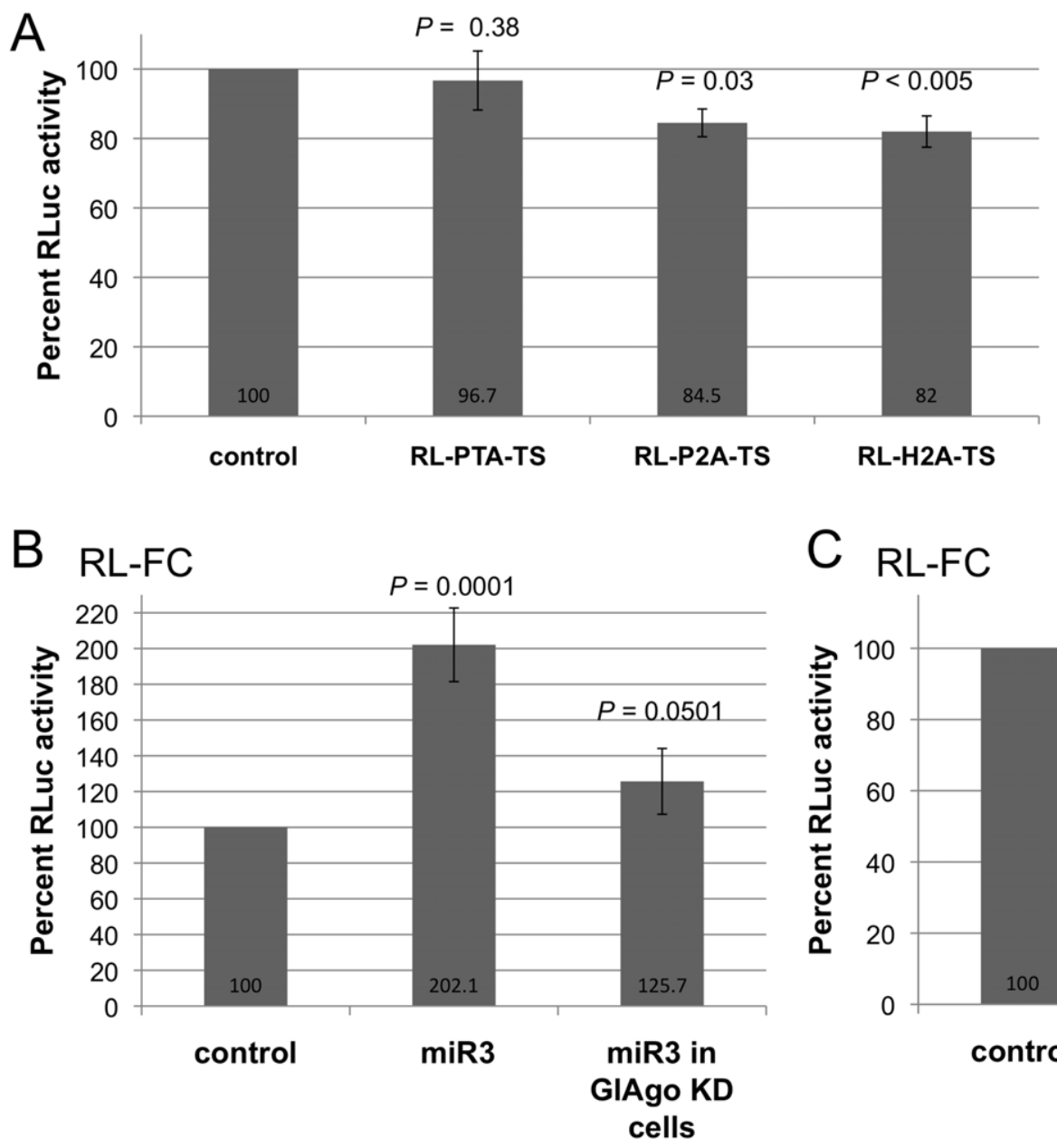

Figure 1. Testing the putative targets of miR3. A) Potential miR3 target sites identified by the miRanda program were tagged to the $3^{\prime}$ UTR of an RLuc transcript and tested against synthetic miR3 in transfected Giardia trophozoites. The three target sites were derived from the $3^{\prime}$-ends of potassium-transporting ATPase (PTA-TS), Ser/Thr phosphatase 2A (P2A-TS) and histone H2A (H2A-TS), respectively. The RLuc activity, reflecting efficiency of miR3 repression, suggests a reversed correlation with the predicted miRanda scores and free energy of binding of the targeting sites (see Table 1). The control represents the RLuc activities expressed by the chimeric transcripts without introducing miR3 into the cells. They were each set at $100 \%$ and presented in a single column as the "control". B) The expression of RL-FC is activated by two-fold in the presence of miR3 in Giardia trophozoites. This activation is significantly reduced in the GIAgo KD cells. The control shows expression of RL-FC without miR3 and is set at $100 \%$. C) Co-transfection of Giardia with an equivalent amount of miR3 2' O-methyl antisense oligo (ASO) and miR3 abolishes the miR3-mediated activation of the RL-FC expression. Transfection with miR3 ASO alone causes a decrease in RLuc activity by $\sim 25 \%$, suggesting that endogenous miR3 is also activating expression of RL-FC. The control indicates expression of RL-FC without ASO or miR3 and is set at $100 \%$. These data represent the mean $+/-$ SD from three independent experiments. The $P$-values were determined using an unpaired student $\mathrm{t}$-test and compared to the control. doi:10.1371/journal.pone.0055672.g001

transcript carrying a fully complementary target in Giardia. We first episomally expressed an N-terminally HA-tagged histone H2A gene carrying its original target site (HA-H2A-TS) under the control of a tetracycline inducible promoter [30]. Two additional mutants were added to the study. One has a portion of the original target site downstream from the stop codon deleted so that the seed sequence of miR3 cannot bind to the truncated target site (HA-H2A). The other has the target site converted to a sequence fully complementary to that of miR3 (HA-H2A-FG) (Figure 2A). Expression of the three constructs in Giardia, monitored by Western blot and immunofluorescence microscopy, showed that HA-H2A was expressed by all three constructs (data not shown).

When synthetic miR3 was introduced into the transfectants, expression of HA-H2A-TS was repressed by $31.8 \%$ (Figure 2B), HA-H2A remained relatively unchanged at $100.7 \%$ (Figure 2C), whereas HA-H2A-FG was enhanced to $203.3 \%$ of the original level (Figure 2D). These results are in good agreement with those from the previously observed RLuc expression (Figure 1). Thus, binding of miR 3 to a fully complementary target site at the $3^{\prime}$ end of H2A mRNA also leads to a 2-fold enhanced expression.

The effects of other miRNAs in Giardia on their respective fully complementary targets. In an effort to understand the mechanism of miR3-mediated activation, we initially looked at the estimated free energy $(\Delta G)$ of miR3 binding to its fully complementary target site, which is $-52.43 \mathrm{kcal} / \mathrm{mole}$. This is very similar to that of miR2 binding $(\Delta \mathrm{G}=-52.22 \mathrm{kcal} /$ mole), miR4 binding $(\Delta \mathrm{G}=-50.29 \mathrm{kcal} / \mathrm{mole})$ [24], miR5 binding $(\Delta \mathrm{G}=-52.42 \mathrm{kcal} / \mathrm{mole}) \quad[22], \quad$ miR6 binding $(\Delta \mathrm{G}=-52.78 \mathrm{kcal} / \mathrm{mole})$ and miR 10 binding $(\Delta \mathrm{G}=-56.14 \mathrm{kcal} / \mathrm{mole})$ to their respective fully complementary targets (Figure S2A). Bindings of miR4 [24] and miR5 [22] to their fully complementary targets have been shown to repress translation to a similar extent as binding to the native target sites [22,24]. The effects of $\mathrm{miR} 2, \operatorname{miR} 6$ and $\mathrm{miR} 10$ on their respective 
Table 1. Three putative targets of miR3 selected from the Giardia genomic database for testing.

\begin{tabular}{|c|c|c|c|c|c|}
\hline $\begin{array}{l}\text { MiRanda } \\
\text { score }\end{array}$ & $\Delta \mathrm{G}$ (kcal/mole) & $\begin{array}{l}\text { Target site } \\
\text { location } \\
\text { (nts) }^{\mathrm{a}}\end{array}$ & Gene ID & $\begin{array}{l}\text { Encoded } \\
\text { protein }\end{array}$ & Target site ${ }^{b}$ \\
\hline \multirow[t]{3}{*}{178} & -33.52 & $38-63$ & GL50803_96670 & $\begin{array}{l}\text { Potassium-transporting } \\
\text { ATPase alpha chain } 1\end{array}$ & $\begin{array}{l}\text { miR3: } 3^{\prime} \text { CCA GUC } \\
\text { UCG CCA CUA CGC } \\
\text { AAC AGA CG }\end{array}$ \\
\hline & & & & & | : : :||| || ||| ||| ||| | \\
\hline & & & & & $\begin{array}{l}5^{\prime} \text { ACU GGU CUU } \\
\text { GGU AAU GCG } \\
\text { UUG UCU GG }\end{array}$ \\
\hline \multirow[t]{3}{*}{177} & -31.62 & $47-73$ & GL50803_7439 & $\begin{array}{l}\text { Ser/Thr phosphatase } 2 \mathrm{~A} \text {, } \\
\text { reg sub A }\end{array}$ & $\begin{array}{l}\text { miR3: 3' CCA GUC UCG } \\
\text { CCA CUA C- GCA } \\
\text { ACA GAC G }\end{array}$ \\
\hline & & & & & | : | ||| | ||| | ||| ||| ||| \\
\hline & & & & & 5' AGU AGG AGC UG- GAT GCC CGU UGU CUG C \\
\hline \multirow[t]{3}{*}{134} & -20.93 & $37-63$ & GL50803_14256 & Histone $\mathrm{H} 2 \mathrm{~A}$ & $\begin{array}{l}\text { miR3: } 3^{\prime} \text { CCA GU- CUC GCC } \\
\text { ACU ACG CA- ACA GAC G }\end{array}$ \\
\hline & & & & & || || | ||| |: || ||| ||| \\
\hline & & & & & $\begin{array}{l}\text { 5' UCG CAG GAU } \\
\text { CUU UGA -GU GUC } \\
\text { UGU CUG U }\end{array}$ \\
\hline
\end{tabular}

a- The numbering is based on the $150 \mathrm{nts}$ chosen for analysis ( $50 \mathrm{nts}$ upstream and $100 \mathrm{nts}$ downstream of the stop codon).
b- Target sites were identified with the miRanda program. The stop codon is shown in bold.

doi:10.1371/journal.pone.0055672.t001

fully complementary targets were tested in the current study. Both miR 6 and miR 10 repressed the expression of target-site carrying RLuc mRNA by $\sim 20 \%$ (Figure S2B). But miR2 enhanced the expression by $\sim 30 \%$ (Figure S2B). Quantitative RT-PCR (RTqPCR) analysis of the reporter mRNAs indicated no significant change in the mRNA levels with the introduction of miRNAs, suggesting translational repression by miR 6 and miR 10 but activation by miR2 (Figure S2G). There are thus two miRNAs in Giardia, miR3 and miR2, showing activation of translation of an mRNA carrying fully complementary target sites.

\section{Mutations of the Fully Complementary Target of miR3}

Successive alterations of the fully complementary target of miR3 were carried out by mutations starting from the 5 '-end of the target (Figure 3A). A disruption of the very first base-pair (RL-FC1) (Figure S3) reduced the miR3-mediated activation by $76.2 \%$ (Figure 3B). Further disruption of the base-pairs in 2 nt increments up to the first $10 \mathrm{nts}$ from the $5^{\prime}$-end of the target site (Figure S3), however, showed only slight variations in the reduced activation. This reduction ranged from between $76.9 \%$ (RL-FC-8) to $94.3 \%$ (RL-FC-6) and is within experimental error (Figure 3B). Thus, full complementation between miR3 and the target throughout the entire 26 base-pairs (bp) is apparently essential for the full 2-fold activation, whereas a residual $\sim 20 \%$ activation is maintained when complementation is disrupted along the first $10 \mathrm{bp}$. A steady linear reduction of the free energy of binding was calculated from -49.36 to $-29.90 \mathrm{kcal} /$ mole upon the stepwise loss of complementation along the $10 \mathrm{bp}$ (Figure 3B), suggesting that free energy of binding is not related to the activating effect of miR3.

Further disruption of base-pairings from the $12^{\text {th }}$ to the $18^{\text {th }} \mathrm{nt}$ from the 5 '-end of the target sequence (Figure S3) showed repression of RLuc expression increasing from 11.2\% (RL-FC-12) to $36.9 \%$ (RL-FC-18) (Figure 3B). This is correlated with the estimated $\Delta \mathrm{G}$ of miR3-target binding from -26.60 to $-13.51 \mathrm{kcal} / \mathrm{mole}$, suggesting that higher free energy of binding leads to more efficient repression. This interesting finding agrees with our previous finding that miR3 exerted little effect on the expression of potassium transporting ATPase alpha chain 1 transcript (see Figure 1A), which has a target site fully complementary to the miR 3 from nt 14 to 25 (Table 1 ).

To verify whether the miR3-mediated repression of RL-FG-18 expression requires GlAgo, the experiment was repeated in the GlAgo KD cells as previously described (see Figure 2B). No apparent effect of miR3 was observed in these cells (data not shown), indicating an essential role of GlAgo.

Further loss of complementation with the miR3 seed sequence in the target abolished all the effect of miR3 on RLuc expression (data not shown), suggesting that full base-pairings between nts 19 and 26 in the target and nts 1 and 8 in miR3 are essential for the repressive function of miR3.

\section{The Seed Sequence of miR3 Alone is Sufficient to Repress Translation}

The maximum repressive effect of miR3 demonstrated on the expression of RL-FC-18 (Figure 3B) indicates that the mere formation of a seed duplex (nt 1 to 8) could be sufficient for maximum translation repression. We chemically synthesized an 8nt oligomer corresponding to the seed sequence (nt 1-8) of miR3 (8-nt Seed). This 8-nt Seed was tested on Giardia cells expressing RL-FG or RL-FG-18 and found to inhibit the RLuc expression by $\sim 25 \%$ (Figure $4 \mathrm{~A}$ ). When the experiment was repeated in GlAgo knockdown cells, the repressive effect was largely abolished (Figure 4B), suggesting the dependence of 8-nt Seed action on GlAgo. When this oligomer was tested on the expression of RLH2A-TS (see Figure 1A), it showed a $\sim 23 \%$ repression (Figure $4 \mathrm{~A}$ ) similar to that observed on RL-FG and RL-FC-18. Thus, an 8-nt Seed oligomer works well on targets of widely varying upstream sequences as long as formation of the seed duplex remains unaffected. 
A

miR3: 3' CCA GU- CUC GCC ACU ACG CA- ACA GAC G

II I| | ||| |: || ||| |||

HA-H2A-TS: 5' UCG CAG GAU CUU UGA -GU GUC UGU CUG U

miR3: 3 , CCA GU- CUC GCC ACU ACG CAA CAG AC

HA-H2A: 5' CGG UCG CAG GAU CUU CUU UGA AAG UAA AGU AAU UAA 3'

miR3: 3'

CC AGU CUC GCC ACU ACG CAA CAG ACG

\| ||||||||||||||||||||||||

HA-H2A-FC: 5' GAU CUU CGG UCA GAG CGG UGA UGC GUU GUC UGC A 3'

B

HA-H2A-TS

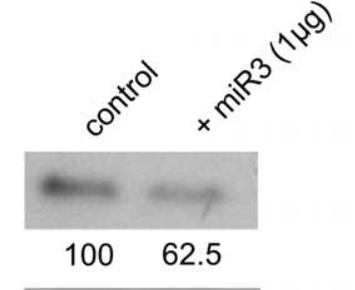

Tubulin
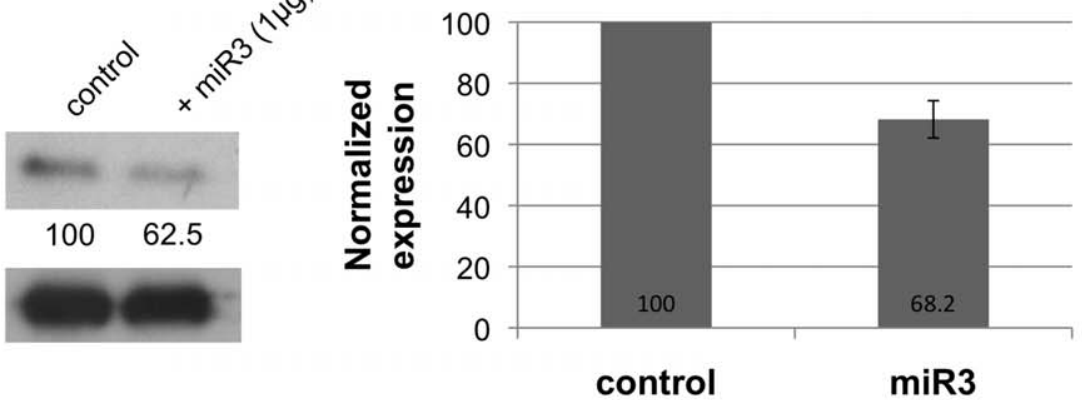

C

$\mathrm{HA}-\mathrm{H} 2 \mathrm{~A}$
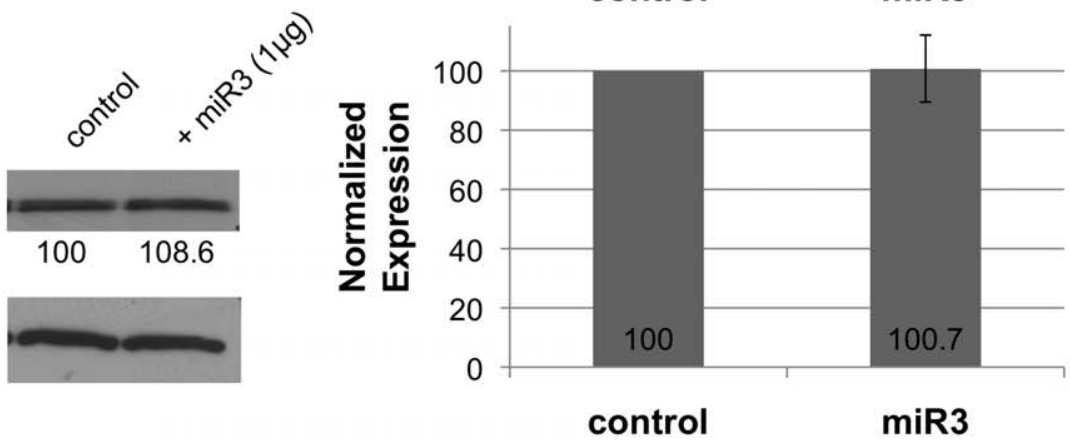

D

Tubulin
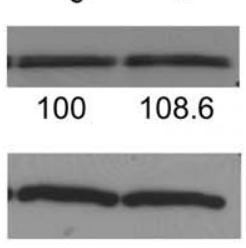

control

miR3
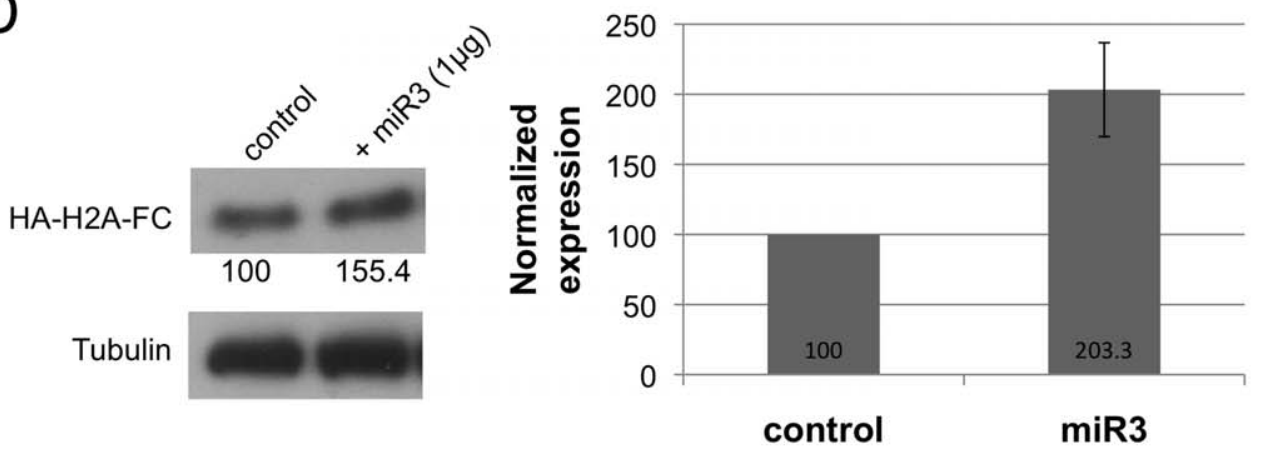

Figure 2. Effects of miR3 on HA-tagged histone H2A expression in Giardia carrying varied target sites. A) A diagram of the three different target sites carried by the HA-H2A transcript showing their base-pairings with miR3. B, C and D) The effects of miR3 on the expression of $\mathrm{HA}-\mathrm{H} 2 \mathrm{~A}$ carrying different target sites were monitored by Western blot (left panel) with the mean intensity $+/-$ SD of individual stained bands from three independent experiments analyzed by densitometry (right panel). B) HA-H2A-TS: C) HA-H2A; D) HA-H2A-FC. The controls in B), C) and D) show the levels of expression of different transcripts without co-introduction with miR3. They are each set at $100 \%$.

doi:10.1371/journal.pone.0055672.g002

Thus, in essence, we have made an interesting observation that miR3 requires only its seed sequence to form a duplex with the target to exert translational repression. The seed sequence itself is capable of performing the repressive function of miR3 without the rest of the 3 '-sequence. The function of this 8-nt Seed oligomer, also mediated by Argonaute, may provide a new approach for us to look at the mechanism of miRNA repression. More intriguingly, we also observed that when miR3 binds to a fully complementary target, it exerts a strong GlAgo-dependent activation of translation instead of a reduction. This is the first time as far as we are aware that a miRNA could exert totally opposite regulatory effects on transcript translation depending solely on the extent of its interaction with the target. 
A

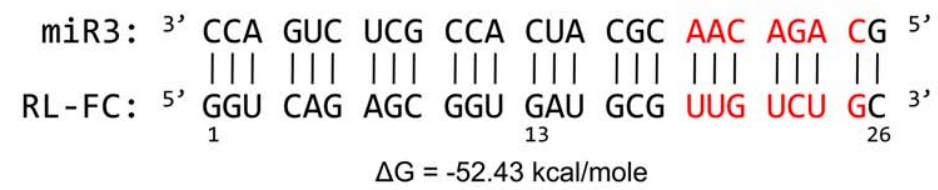

B

$\Delta \mathrm{G}=-52.43 \mathrm{kcal} / \mathrm{mole}$

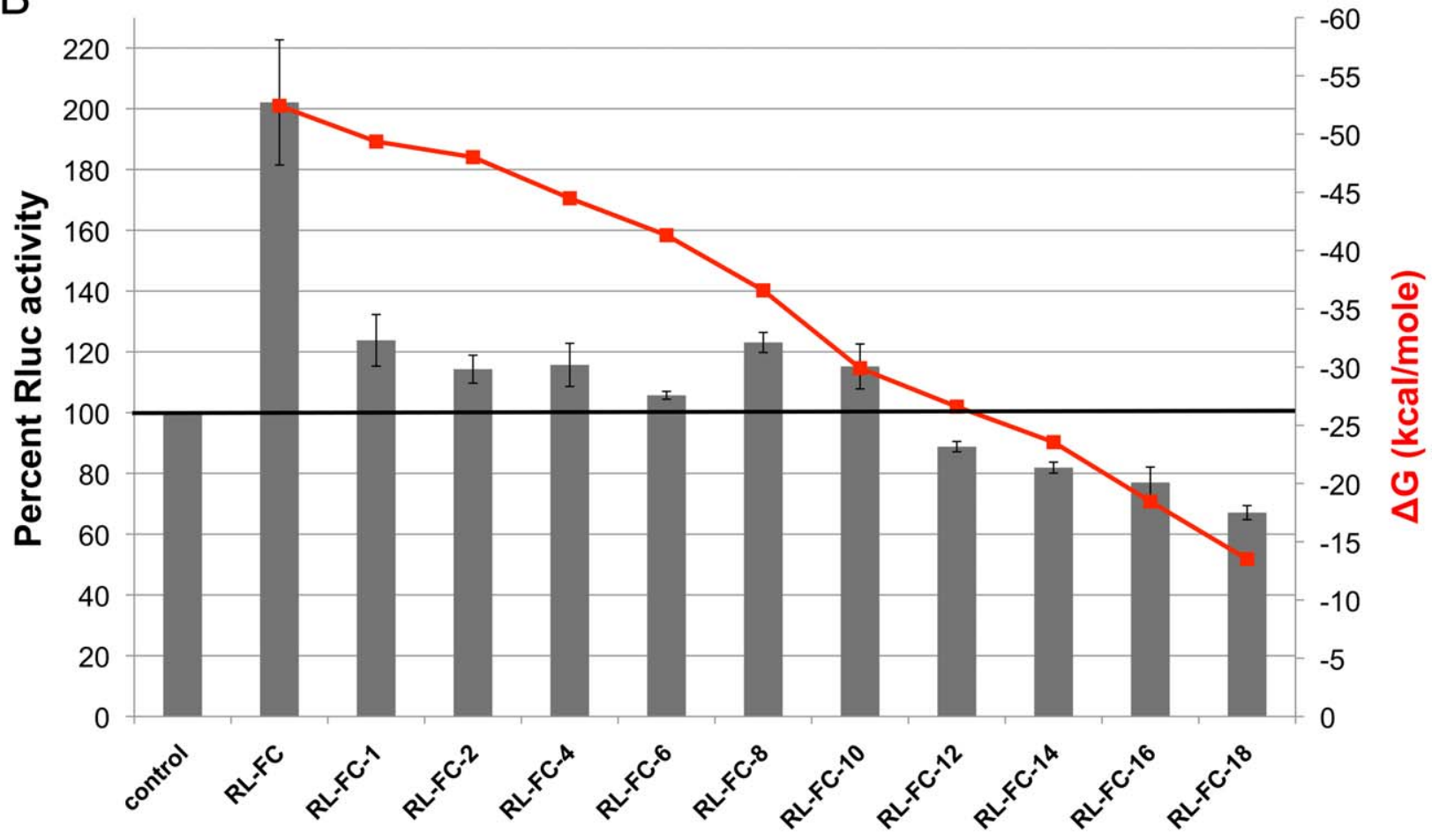

Figure 3. Analysis of mutations to a fully complementary (FC) miR3 target. A) The numberings of nucleotides in FC with the seed sequence shown in red. The $\Delta \mathrm{G}$ derived from base-pairings with miR3 is shown below. B) Mutations of RL-FC were carried out stepwise from the 5'-end of FC to reduce the base-pairings with miR3. Mutant transcripts were transfected into Giardia trophozoites along with miR3 and assayed for RLuc activity. The mean +/- SD from three independent experiments is presented. The $\Delta \mathrm{G}$ values estimated from base-pairings with miR3 for each mutant is shown in an orange-colored line. The control column on the left represents the level of expression of each of the mutant transcripts in the absence of introduced miR3. They are each set at $100 \%$ and presented in a single column. doi:10.1371/journal.pone.0055672.g003

\section{Discussion}

Here we present the first evidence of a biphasic miRNA that can either repress or activate translation based solely on the extent of its base-pairings with the target. Both actions are dependent on the presence of an Argonaute protein, GlAgo, suggesting formation of a functional RNA-induced silencing complex (RISG) including the miRNA, the target and GlAgo for both actions.

\section{The Repressive Function of miR3}

The miR3 mediated translational repression of histone $\mathrm{H} 2 \mathrm{~A}$ in Giardia raises the question on potential global effect of miR3 on chromosomal remodeling. Basic chromatin fibers were found in typical nucleosomal conformations in Giardia trophozoites [31]. They consist of four core histones H1, H2B, H2A and H4 [31]. But extensive chromosome rearrangements [32] and genome plasticity among many of the chromosomes [33] have made investigation of chromosomal remodeling difficult at the present time.

A maximum $36.9 \%$ repression of translation is achieved when only the $8 \mathrm{nt}$ seed sequence in miR3 hybridizes to the target (RLFG-18). It strongly suggests that the sequence downstream from the seed region in miR3 is not required for its repressive function.
When an 8-nt oligomer corresponding to the miR 3 seed sequence was introduced into Giardia cells, a similar GlAgo-dependent repression of $\sim 25 \%$ on the expression of RL-FC-18 or RL-H2ATS was observed (Figure 4). These observations raise an interesting possibility that only the seed sequence of a miRNA and the Argonaute protein is required to form a complex with the target to repress translation of a transcript in Giardia and that an 8nt oligomer is sufficient to interact with GlAgo.

Previous studies have shown that a 9 nt guide DNA complexed with the Thermus thermophilus Argonaute protein was sufficient to promote target RNA cleavage in vitro [34]. A $2.6 \AA$ crystal structure of the PAZ domain from human Argonaute (hAgo) complexed with a 9 bp RNA duplex with a $3^{\prime}-2$ nt overhang in the DNA guide strand was recently analyzed [35]. These data indicate that a small RNA fragment is capable of binding to the Argonaute and forming a duplex with the target. But there has not yet been any previous indication, to our knowledge, that the seed fragment of a miRNA is capable of performing the full function of a miRNA in cells. The current observation could be the first of such an example. It could be a specific phenomenon confined to the ancient protozoan Giardia, which may cast some interest in the evolutionary aspect of miRNA function. Or, if it turns out to be 
A

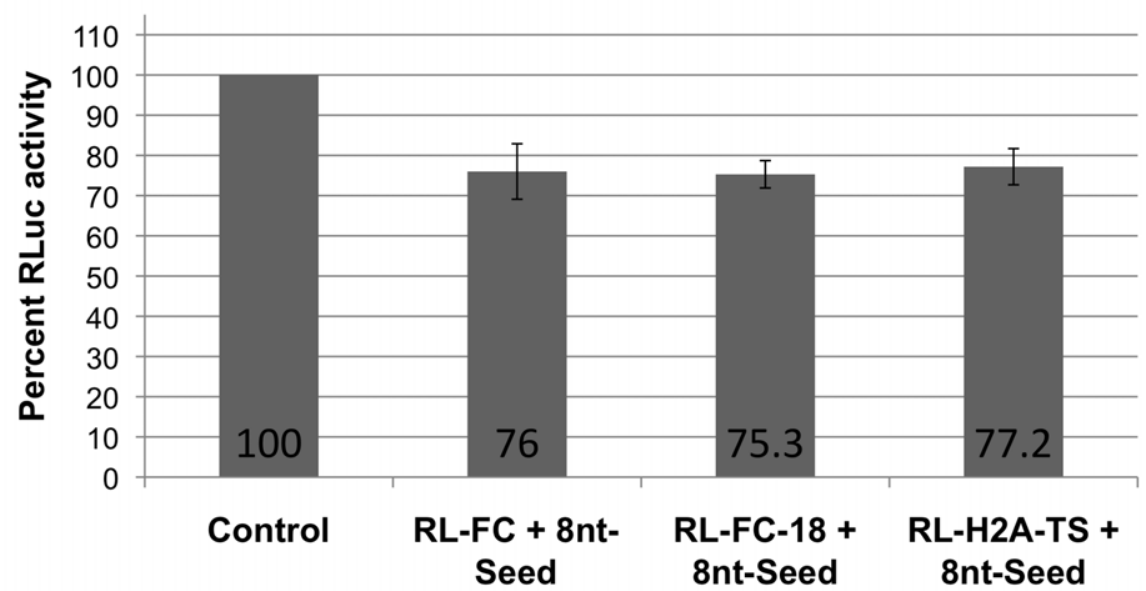

B

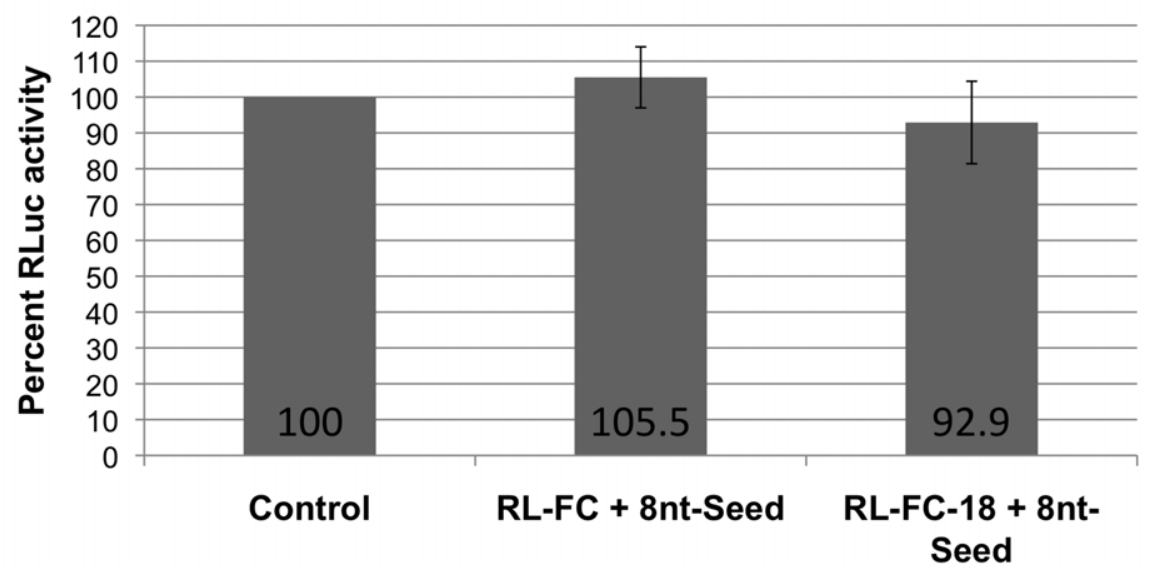

Figure 4. Repression of RLuc transcript expression by the miR3 seed oligomer (8 nt-Seed). A) An 8 nt miR3 seed oligomer (8 nt-Seed) is capable of repressing expression of RL-FC by $24 \%$, RL-FC- 18 by $24.7 \%$ and RL-H2A-TS by $22.8 \%$. B) The 8 nt-Seed was also tested on RL-FC and RL-FC18 expression in the GIAgo KD cells, and showed no apparent effect. The data represent the mean +/- SD from at least two independent assays. The controls show the levels of expression of various chimeric transcripts in the absence of exogenously introduced 8 nt-Seed. The values are set at $100 \%$ and presented in a single control column.

doi:10.1371/journal.pone.0055672.g004

true in other organisms as well, it may shine new lights on our current thinking of the mechanism of miRNA action.

\section{The Activating Function of miR3}

There have been many examples showing that a small RNA could function as either a small interfering (si)RNA or a miRNA depending on the extent of base-pairings with the target [36]. A single splicing Argonaute protein could thus fulfill the role in an RISC for both siRNA and miRNA actions. Crystallographic data shows that the presence of a mismatch at positions 10 or 11 in the miRNA-target duplex places a bulge near the catalytic DDE triad in the PIWI domain of $\mathcal{T}$. thermophilus Argonaute protein, resulting in resistance of the target RNA to Argonaute slicing [34]. The existence of non-slicing Argonautes has been nevertheless demonstrated. In humans, only hAgo2 is capable of slicing mRNA $[37,38]$, even though the conserved DDE catalytic triad is present in hAgol, hAgo2 as well as hAgo3 [37]. In a recent report, the crystal structure of Kluyveromyces polysporus Argonaute was shown to possess a hydrogen-bond network that stabilizes an expanded and repositioned loop L2, inserting a glutamate into the catalytic pocket [39]. This insertion of a glutamate finger apparently completes a catalytic tetrad, activating the Argonaute protein for
RNA cleavage [39]. This particular structural feature could distinguish the slicing from the non-slicing type of Argonaute.

GlAgo appears to possess the DDE triad, but has not shown any mRNA slicing activity suggesting that GlAgo may be missing the structure to form the catalytic tetrad for RNA slicing [22,24,39]. Using mRNAs containing fully complementary target sites, none of the corresponding miRNAs demonstrated any RNAi function (Figure S2B). miR4, miR5, miR6 and miR10 repressed translation to similar extents as those with the imperfect native target sites, whereas miR2 and miR3 stimulated translation when the target sites were fully complementary. The basis behind these opposite actions of miRNAs in Giardia remains unclear. A human miRNA miR-326 has recently been found to act on the predicted target site in HIV genome and decrease the viral replication [40]. When the degree of complementarity between the miRNA and the viral target increases, the extent of repression of HIV replication also increases, thus providing an example contrary to what we have observed.

Examples of miRNA-enhanced gene expression have been recently observed in other organisms. An increasing number of studies have indicated that miRNAs can stimulate posttranscriptional gene expression regulated by the RNA sequence associated 
with micro-ribonucleoproteins [41]. Notably, human miR369-3 was found to activate translation of TNF- $\alpha$ mRNA during cell cycle arrest, but repress the same translation in proliferating cells without affecting the mRNA levels $[42,43]$. The target site is near an AU-rich element and does not form perfect base pairings with miR369-6. The oscillating functions of this miRNA could be attributed to the changing availability of factors required for the opposing effects in different phases of cell development. The liverspecific miR122 binds to two consecutive target sites in the $5^{\prime}$ UTR of hepatitis $\mathrm{G}$ virus RNA and stimulates expression of the viral RNA without affecting its level $[44,45]$. Only the seed region and nts 14-16 in the miRNA are required for base-pairings with the target for the activating function [44]. The reason why target sites located at the 5'-UTR of hepatitis $\mathrm{C}$ virus transcript can mediate the stimulatory function of a miRNA is attributed to the presence of an internal ribosome entry site instead of a cap at the 5 '-UTR of the viral transcript. Thus, though the more detailed mechanisms of action are still unclear, it is apparent that these two examples of translation activation by miRNAs are associated with specific temporal or spatial circumstances and not dependent on full base pairings with the target.

An Argonaute protein is composed of an amino-terminal $\mathrm{N}$ and PAZ domains and carboxy-terminal Mid and PIWI domains. The crystal structure of an Argonaute Asn546 catalytically inactive mutant protein from T. Thermophilus was recently analyzed [46]. Since the mutant protein has lost its slicing activity, a stable ternary complex of the protein with a 5'-phosphorylated $21 \mathrm{nt}$ guide DNA and a perfectly complementary 19 nt target RNA was formed and examined at $2.8 \AA$ resolution. The guide-target duplex starting from the seed region and spanning from position 2 to 16 in a Watson-Crick A-form helical structure was anchored in the Mid pocket with the scissile phosphate between nts 10 and 11 in the target strand positioned opposite to the catalytic residues DDE in the PIWI domain as anticipated. The $\mathrm{N}$ domain, however, blocked propagation of the duplex structure beyond bp16, causing apparent separation between the guide and target strands at this point. The maximum length of the guide-target duplex in the $T$. thermophilus Argonaute crystal structure is thus limited to $16 \mathrm{bp}$. Eukaryotic Argonautes are capable of binding to longer RNA duplexes. Recently, the full-length crystal structure of hAgo2 was resolved [47]. It positions the $\mathrm{N}$ domain away from the central grove and thus allows for a more extended RNA duplex to bind [47]. A similar observation of $K$. polysporus Ago also indicated a lengthening and slight widening of the duplex-binding channel by pushing the $\mathrm{N}$ domain aside to accommodate a longer RNA duplex [39].

To gain a better understanding of the potential interaction between a miR3 duplex and GlAgo, we used homology modeling to predict a potential structure of GlAgo. The relatively low sequence homology between GlAgo and the other available Argonaute crystal structures led us to use three different modeling servers; the I-TASSER homology-modeling server [48], the Phyre2 and the ModWeb servers [49,50]. The three predicted structures of GlAgo were all based on the T. thermophilus Ago crystal structure and appear similar (Figure S4A), providing some confidence in this predicted structure. The predicted structure also agrees well with the hAgo2 crystal structure [47] with the N, PAZ, Mid and PIWI domains structurally well conserved (Figure S4A). The Mid and PIWI domains showed the most structural conservation among the models while the $\mathrm{N}$ and PAZ domains are less conserved probably due to the lower sequence homology (Figure S4A).

The I-TASSER model of GlAgo overlaps well with the crystal structure of T. thermophilus Ago (Figure S4B) [46,48]. A 26 bp RNA duplex was modeled into the GlAgo structure using the crystal structure of T. thermophilus Argonaute Asn546 mutant guide-target duplex as a guide [46]. The duplex structure is anchored in the Mid pocket and flanked by the PAZ and PIWI domains of GIAgo as in the T. Thermophilus Ago (Figure S4G). The $\mathrm{N}$ domain in GlAgo is connected to the PAZ domain by a single loop, suggesting that the $\mathrm{N}$ region in GlAgo is mobile. A presumed movement of the $\mathrm{N}$ domain away from the central grove like that in hAgo2 and $K$. polysporus Ago would enable GlAgo to accommodate the $26 \mathrm{bp}$ duplex (Figure S4C). This modeling could explain how the miRNAs, which have a relatively large size of 26-28 nts and form fully complementary 26-28 nt miRNAtarget duplexes can be accommodated into GlAgo. We thus postulate that, in Giardia, the fully complementary 26 bp miR3target duplex (and probably to a lesser extent the miR2 duplex) may differ from the other miRNA duplexes in interacting with the mobile $\mathrm{N}$ domain and induce specific structural changes in GlAgo. These changes may allow the binding of additional specific proteins to the RISG complex leading to translational activation. Other partial or mismatched miRNA-target duplexes may lead to different conformational changes of GlAgo, resulting in a protein complex for translational repression. A close examination of the sequences of these miRNA duplexes listed in Figure S2A, however, did not immediately explain how the duplexes of miR 2 and miR3 may differ structurally from the rest. The detailed mechanism of miR3-mediated activation of translation remains thus unexplained at this time.

\section{Materials and Methods}

\section{Incorporation of miR3 Target Sites into the RLuc Reporter Gene}

Incorporation of different target sites or target site mutants was done by PCR amplification of the RLuc reporter gene. Two copies of each mutated target site were incorporated into a reverse primer (see Table S2). This reverse primer along with a forward primer containing a T7 promoter (T7 promoter Rluc) was used to PCRamplify the RLuc reporter gene containing a target site in the $3^{\prime}$ UTR. The PCR product was gel purified and used for a second round of PCR to add a poly (A) tail using the T7 promoter Rluc forward primer and the miR 3 comp add polyA $\mathrm{R}$ reverse primer. The resulting PCR product was phenol/chloroform extracted, ethanol precipitated, suspended in sterile water and then sequenced. The purified PCR product was used for capped in vitro transcription using the mMessage mMachine kit (Ambion) following the manufacturer directions. The transcribed RNA was precipitated with $\mathrm{LiCl}$, resuspended in RNase-free water, and quantitated using a Nanodrop 2000 (Thermo Scientific).

\section{miRNA Assay}

The assay for miRNA repression of RLuc transcript expression was performed as previously described using either Giardia WB wild-type or the GlAgo KD WB trophozoites [25]. Giardia WB trophozoites, grown in modified TYI-S-33 media to a density of $10^{7}$ per $\mathrm{ml}$, were pelleted. Cells were washed once in phosphate buffered saline (PBS), once in electroporation buffer $(10 \mathrm{mM}$ K2HPO4-KH2PO4 (pH 7.6), 25 mM HEPES (free acid), $120 \mathrm{mM} \mathrm{KCl}, 0.15 \mathrm{mM}$ CaCl2, 2 mM EGTA, 5 mM MgCl2, $2 \mathrm{mM}$ ATP, $4 \mathrm{mM}$ Glutathione), and finally suspended in electroporation buffer. Reporter mRNA $(\sim 3 \mu \mathrm{g})$, yeast tRNA $(125 \mu \mathrm{g})$, and, if needed, $1 \mu \mathrm{g}$ of $5^{\prime}$-phosphate-miR3 RNA (miR3) (IDT) were added to the cell suspension $\left(\sim 10^{6}\right.$ cells), incubated on ice for $10 \mathrm{~min}$ and then subjected to electroporation using a BioRad Gene Pulser Xcell (Voltage: $450 \mathrm{~V}$, Capacitance: $500 \mu \mathrm{F}$, 
Resistance: $\infty$ ). Cells were then incubated on ice for $10 \mathrm{~min}$ and added to pre-warmed culture medium. The transfected cells were incubated at $37^{\circ} \mathrm{C}$ for 5 hours, pelleted, washed once in PBS, and lysed using the Renilla luciferase assay kit (Promega). The lysate was centrifuged at $13,000 \mathrm{~g}$ for $2 \mathrm{~min}$ to remove cellular debris. The cleared lysate was used to test for Renilla luciferase activity. The protein concentration of the cleared lysate was measured using the Bradford assay (Bio-Rad) and used to normalize the total protein concentration.

\section{HA-H2A-TS Cloning and Expression}

The HA-tagged histone $\mathrm{H} 2 \mathrm{~A}$ gene with its putative target site (TS) for miR3 and the mutant constructs were PCR amplified from Giardia genomic DNA using the HA-H2A F primer and the appropriate reverse primer (see Table S2). The product was cloned into pJet2.1 (Fermentas), sequenced and sub-cloned into the pNlop4 vector, a derivative of the pNLop3-GtetR vector, using $\mathcal{N} c o \mathrm{I}$ and $E_{c o R} \mathrm{I}$ [30]. The resulting construct was transfected into Giardia trophozoites as previously described and the transfectants

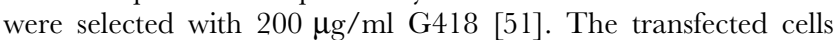
were induced with $10 \mu \mathrm{g} / \mathrm{ml}$ of tetracycline at $37^{\circ} \mathrm{C}$ for 24 hours and assayed by Western blot to confirm expression of the protein.

\section{Supporting Information}

Figure S1 Characterization of miR3. A) The FISH assay using the $5^{\prime}$-end $26 \mathrm{nt}$ and $3^{\prime}$-end $26 \mathrm{nt}$ sequences of the snoRNA GlsR 16 as probes [25] indicates that the snoRNA is predominately localized to the nucleolus with some presence in the nucleus. miR3 is primarily localized to the cytoplasm of Giardia trophozoites. B) qRT-PGR indicates that miR3 is enriched in the small RNA band co-immunoprecipitated with HA-GlAgo [24].

(TIF)

Figure S2 Effects of all six identified Giardia miRNAs on translation of RLuc mRNAs carrying fully complementary target sites. A) Alignment of the six miRNAs with their respective fully complementary targets. B) Effects of the six miRNAs on expression

\section{References}

1. Bartel DP (2009) MicroRNAs: target recognition and regulatory functions. Cell 136: 215-233.

2. Pasquinelli AE (2012) MicroRNAs and their targets: recognition, regulation and an emerging reciprocal relationship. Nat Rev Genet 13: 271-282.

3. Bazzini AA, Lee MT, Giraldez AJ (2012) Ribosome profiling shows that miR430 reduces translation before causing mRNA decay in zebrafish. Science 336: 233-237.

4. Djuranovic S, Nahvi A, Green R (2012) miRNA-mediated gene silencing by translational repression followed by mRNA deadenylation and decay. Science 336: $237-240$.

5. Abbott AL, Alvarez-Saavedra E, Miska EA, Lau NC, Bartel DP, et al. (2005) The let-7 MicroRNA family members mir-48, mir-84, and mir-241 function together to regulate developmental timing in Caenorhabditis elegans. Dev Cell 9: 403-414.

6. Chen CZ, Li L, Lodish HF, Bartel DP (2004) MicroRNAs modulate hematopoietic lineage differentiation. Science 303: 83-86.

7. Giraldez AJ, Cinalli RM, Glasner ME, Enright AJ, Thomson JM, et al. (2005) MicroRNAs regulate brain morphogenesis in zebrafish. Science 308: 833-838.

8. Chen JF, Mandel EM, Thomson JM, Wu Q, Callis TE, et al. (2006) The role of microRNA-1 and microRNA-133 in skeletal muscle proliferation and differentiation. Nat Genet 38: 228-233.

9. Brennecke J, Hipfner DR, Stark A, Russell RB, Cohen SM (2003) bantam encodes a developmentally regulated microRNA that controls cell proliferation and regulates the proapoptotic gene hid in Drosophila. Cell 113: 25-36.

10. Calin GA, Croce CM (2006) MicroRNA signatures in human cancers. Nat Rev Cancer 6: 857-866.

11. Lu J, Getz G, Miska EA, Alvarez-Saavedra E, Lamb J, et al. (2005) MicroRNA expression profiles classify human cancers. Nature 435: 834-838.

12. Cullen BR (2006) Viruses and microRNAs. Nat Genet 38 Suppl: S25-30.

13. Russo A, Potenza N (2011) Antiviral effects of human microRNAs and conservation of their target sites. FEBS Lett 585: 2551-2555. of RLuc carrying their respective fully complementary target sites. C) qRT-PCR estimation of the levels of RLuc mRNAs following the actions of miRNAs. None of the mRNAs showed signs of reduced level, suggesting that Giardia miRNAs act on translation of the mRNAs.

(TIF)

Figure S3 Sequences of different FG target mutants used in this study. Red letters indicate the altered nucleotides and green letters specify the seed sequence in the target.

(TIF)

Figure S4 Homology modeling of GlAgo. A) The ModWeb, Phyre2 and I-TASSER models of GlAgo based on the crystal structure of T. thermophilus Ago are presented [48,49,50]. An ITASSER model of GlAgo based on the crystal structure of hAgo2 is also included $[47,48]$. All four models bear excellent similarities. The model analysis and image presentation was performed with UCSF Chimera [52]. B) The I-TASSER model of GlAgo overlapped with the crystal structure of T. thermophilus Ago (3HK2) [48]. C) Modeling of a 26 bp RNA duplex into the ITASSER model of GlAgo required a slight movement of the $\mathrm{N}$ domain toward the right to accommodate the inserted duplex. Movement of the $\mathrm{N}$ domain and modeling of the $26 \mathrm{nt}$ RNA duplex were performed with UCSF Chimera [52].

(TIF)

Table S1 List of miRanda identified putative miR3 target sites in Giardia.

(XLSX)

Table S2 List of PGR primers.

(XLSX)

\section{Author Contributions}

Conceived and designed the experiments: AAS CCW. Performed the experiments: AAS WL. Analyzed the data: AAS WL CCW. Wrote the paper: AAS CCW.

14. Brennecke J, Stark A, Russell RB, Cohen SM (2005) Principles of microRNAtarget recognition. PLoS Biol 3: e85.

15. Brodersen P, Voinnet $\mathrm{O}$ (2009) Revisiting the principles of microRNA target recognition and mode of action. Nat Rev Mol Cell Biol 10: 141-148.

16. Lewis BP, Burge CB, Bartel DP (2005) Conserved seed pairing, often flanked by adenosines, indicates that thousands of human genes are microRNA targets. Cell 120: $15-20$.

17. Hutvagner G, Zamore PD (2002) A microRNA in a multiple-turnover RNAi enzyme complex. Science 297: 2056-2060.

18. Yekta S, Shih IH, Bartel DP (2004) MicroRNA-directed cleavage of HOXB8 mRNA. Science 304: 594-596.

19. Brodersen P, Sakvarelidze-Achard L, Bruun-Rasmussen M, Dunoyer P, Yamamoto YY, et al. (2008) Widespread translational inhibition by plant miRNAs and siRNAs. Science 320: 1185-1190.

20. Umbach JL, Kramer MF, Jurak I, Karnowski HW, Coen DM, et al. (2008) MicroRNAs expressed by herpes simplex virus 1 during latent infection regulate viral mRNAs. Nature 454: 780-783.

21. Wu L, Fan J, Belasco JG (2008) Importance of translation and nonnucleolytic ago proteins for on-target RNA interference. Curr Biol 18: 1327-1332.

22. Li W, Saraiya AA, Wang CC (2011) Gene regulation in Giardia lambia involves a putative microRNA derived from a small nucleolar RNA. PLoS Negl Trop Dis 5: e1338.

23. Li W, Saraiya AA, Wang CC (2012) The profile of snoRNA-derived microRNAs that regulate expression of variant surface proteins in Giardia lamblia. Cell Microbiol 14: 1455-1473.

24. Saraiya AA, Li W, Wang CC (2011) A microRNA derived from an apparent canonical biogenesis pathway regulates variant surface protein gene expression in Giardia lamblia. RNA 17: 2152-2164.

25. Saraiya AA, Wang CC (2008) snoRNA, a novel precursor of microRNA in Giardia lamblia. PLoS Pathog 4: e1000224.

26. Macrae IJ, Zhou K, Li F, Repic A, Brooks AN, et al. (2006) Structural basis for double-stranded RNA processing by Dicer. Science 311: 195-198. 
27. John B, Enright AJ, Aravin A, Tuschl T, Sander C, et al. (2004) Human MicroRNA targets. PLoS Biol 2: e363.

28. Aurrecoechea C, Brestelli J, Brunk BP, Carlton JM, Dommer J, et al. (2009) GiardiaDB and TrichDB: integrated genomic resources for the eukaryotic protist pathogens Giardia lamblia and Trichomonas vaginalis. Nucleic Acids Res 37: D526-530.

29. Horwich MD, Zamore PD (2008) Design and delivery of antisense oligonucleotides to block microRNA function in cultured Drosophila and human cells. Nat Protoc 3: 1537-1549.

30. Sun CH, Tai JH (2000) Development of a tetracycline controlled gene expression system in the parasitic protozoan Giardia lamblia. Mol Biochem Parasitol 105: 51-60.

31. Triana O, Galanti N, Olea N, Hellman U, Wernstedt C, et al. (2001) Chromatin and histones from Giardia lamblia: a new puzzle in primitive eukaryotes. J Cell Biochem 82: 573-582.

32. Le Blancq SM (1994) Chromosome rearrangements in Giardia lamblia. Parasitol Today 10: 177-179.

33. Upcroft JA, Chen N, Upcroft P (1996) Mapping variation in chromosome homologues of different Giardia strains. Mol Biochem Parasitol 76: 135-143.

34. Wang Y, Juranek S, Li H, Sheng G, Tuschl T, et al. (2008) Structure of an argonaute silencing complex with a seed-containing guide DNA and target RNA duplex. Nature 456: 921-926.

35. Ma JB, Ye K, Patel DJ (2004) Structural basis for overhang-specific small interfering RNA recognition by the PAZ domain. Nature 429: 318-322.

36. Doench JG, Petersen CP, Sharp PA (2003) siRNAs can function as miRNAs. Genes Dev 17: 438-442.

37. Liu J, Carmell MA, Rivas FV, Marsden CG, Thomson JM, et al. (2004) Argonaute2 is the catalytic engine of mammalian RNAi. Science 305: 14371441 .

38. Meister G, Landthaler M, Patkaniowska A, Dorsett Y, Teng G, et al. (2004) Human Argonaute 2 mediates RNA cleavage targeted by miRNAs and siRNAs. Mol Cell 15: 185-197.
39. Nakanishi K, Weinberg DE, Bartel DP, Patel DJ (2012) Structure of yeast Argonaute with guide RNA. Nature 486: 368-374.

40. Houzet L, Klase Z, Yeung ML, Wu A, Le SY, et al. (2012) The extent of sequence complementarity correlates with the potency of cellular miRNAmediated restriction of HIV-1. Nucleic Acids Res 40: 11684-11696.

41. Vasudevan S (2012) Posttranscriptional upregulation by microRNAs. Wiley Interdiscip Rev RNA 3: 311-330.

42. Vasudevan S, Steitz JA (2007) AU-rich-element-mediated upregulation of translation by FXR1 and Argonaute 2. Cell 128: 1105-1118.

43. Vasudevan S, Tong Y, Steitz JA (2007) Switching from repression to activation: microRNAs can up-regulate translation. Science 318: 1931-1934.

44. Roberts AP, Lewis AP, Jopling CL (2011) miR-122 activates hepatitis G virus translation by a specialized mechanism requiring particular RNA components. Nucleic Acids Res 39: 7716-7729.

45. Henke JI, Goergen D, Zheng J, Song Y, Schuttler CG, et al. (2008) microRNA122 stimulates translation of hepatitis C virus RNA. EMBO J 27: 3300-3310.

46. Wang Y, Juranek S, Li H, Sheng G, Wardle GS, et al. (2009) Nucleation, propagation and cleavage of target RNAs in Ago silencing complexes. Nature 461: 754-761

47. Schirle NT, Macrae IJ (2012) The Crystal Structure of Human Argonaute2. Science 336: 1037-1040

48. Roy A, Kucukural A, Zhang Y (2010) I-TASSER: a unified platform for automated protein structure and function prediction. Nat Protoc 5: 725-738.

49. Eswar N, Eramian D, Webb B, Shen MY, Sali A (2008) Protein structure modeling with MODELLER. Methods Mol Biol 426: 145-159.

50. Kelley LA, Sternberg MJ (2009) Protein structure prediction on the Web: a case study using the Phyre server. Nat Protoc 4: 363-371.

51. Singer SM, Yee J, Nash TE (1998) Episomal and integrated maintenance of foreign DNA in Giardia lamblia. Mol Biochem Parasitol 92: 59-69.

52. Pettersen EF, Goddard TD, Huang CC, Couch GS, Greenblatt DM, et al. (2004) UCSF Chimera-a visualization system for exploratory research and analysis. J Comput Chem 25: 1605-1612. 